\title{
The International Space Station As a Free Flyer Servicing Node
}

\author{
Jeffrey Antol ${ }^{1}$, David E. Headley ${ }^{2}$ \\ ${ }^{I}$ NASA, Spacecraft and Sensors Branch/Space Systems and Concepts Division, NASA Langley Research Center, \\ Mail Stop 328, Hampton,VA 23681-2199, Phone: 757-864-5804, Fax: 757-827-9061, j.antol@larc.nasa.gov \\ ${ }^{2}$ The Boeing Company, Advanced Product Research and Development, 100 McDonnell Douglas Way, \\ Titusville, Florida 32780, Phone: 407-383-2822, Fax: 407-269-6208, david.e.headley@ boeing.com
}

\begin{abstract}
The International Space Station will provide a multitude of opportunities for an expanding customer base to make use of this international resource. One such opportunity is servicing of various visiting vehicles that are in a similar orbit to the station. Servicing may include change-out of payloads, replenishment of consumables, repair, and refurbishment operations. Previous studies have been conducted in which "paper" free flyers have been assessed against the station's ability to accommodate them. Over the last several months though, an already flown free flyer, EURECA, was assessed as a real-life visiting free flyer design reference mission. Issues such as capture/berthing, servicing, logistics support, and stowage were assessed for station design and operational approaches. This paper will highlight critical visiting vehicle design considerations, identify station issues, and provide recommendations for accommodation of a wide range of visiting vehicle requirements of the future.
\end{abstract}

\section{EURECA AS AN ISS-SUPPORTED FREE FLYER}

The International Space Station (ISS), once operational, may provide a capability to service various visiting vehicles (VV) that are in a relatively similar orbit to the station. Servicing of these vehicles (free-flying spacecraft) would include change-out of payloads, replenishment of consumables, repair, and refurbishment operations. A number of free-flying platforms, existing and planned, could potentially use this capability (see Figure 1).

The EURECA platform (Daimler-Benz Aerospace, 1996), an existing free flyer (see Figure 2), was assessed against the station's ability to accommodate free-flyer servicing. EURECA provides a test case that covers a full range of free flyer servicing needs. Designed as manned system compatible (i.e., launched from the shuttle), EURECA provides proven hardware and operations. Its untended mission duration classifies it as an ISS "visiting vehicle". Also, commercial marketing of EURECA's capabilities has indicated an interest in a co-orbiting platform for ISS, long-duration experiment operations, routine product processing and missions within the ISS orbit.

\begin{tabular}{|l|c|c|c|c|c|}
\hline \multirow{2}{*}{ Capabilitv } & Spartan 201 & Spartan 400 & LM 700 & Astro Spas & EURECA \\
\hline Primary Mission & Astronomy & All Science & Communications & General Sciences & Microgravity \\
\hline Mission Duration & $40 \mathrm{hrs}$ & $12 \mathrm{mo}$ & $84 \mathrm{mo}$ & $1 / 2 \mathrm{mo}$ & $18 \mathrm{mo}$ \\
\hline Payload Mass (kg) & 1,136 & 2,000 & 500 & 2,000 & 1,000 \\
\hline Active Cooling & Yes & No & Yes & No & Yes \\
\hline Propellants & Cold Gas & Hydrazine & Hydrazine & $\mathrm{N}_{2}$ or He & Hydrazine, $\mathrm{N}_{2}$ \\
\hline
\end{tabular}

FIGURE 1. Example Space Platforms. 


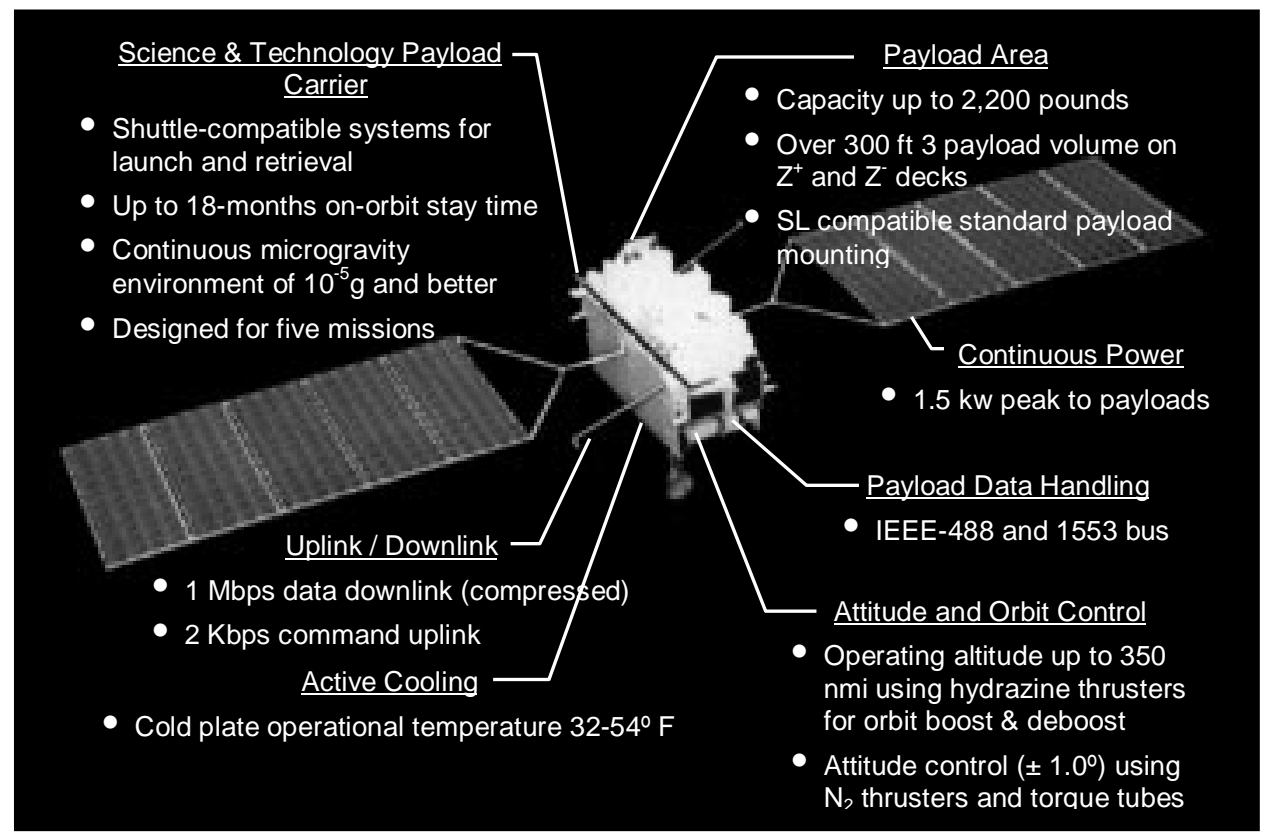

FIGURE 2. EURECA Hardware Overview.

\section{EURECA Requirements and Design Impacts}

Servicing EURCA on-orbit, as opposed to returning it to the Earth, presents a number of changes to the EURECA requirements and impacts the EURECA vehicle design in several areas:

Payload Change-Out. EURECA structural, plumbing, electrical, command/data interfaces to the payloads would have to be changed. The Express Pallet Adapter (EPA) hardware, which allows on-orbit change-out of attached ISS payloads could potentially be adapted for use on EURECA. Also, automated payload-to-EURECA interface tests would be needed for verification following installation of a payload.

Berthing of EURECA to ISS. If the EURECA were to be berthed at an attached payload location for servicing operations, it would have to accommodate the passive side of Payload Attach System (PAS). The PAS provides a structural interface to ISS as well as power and data interfaces. Re-supply of propellant and gases would have to be accomplished through robotic or Extra Vehicular Activity (EVA) operations which would require automated/EVA compatible fluids interface panels on the EURECA.

Attitude and Orbit Control Systems Enhancement. EURECA's systems will need to accommodate automated and ISS control for rendezvous and capture operations. This may be accomplished through additional cold gas system elements, accommodation of GPS equipment, and changes to EURECA electronics and software.

Logistics Re-Supply - Payloads and EURECA. A logistics carrier will be needed to carry EURECA re-supply and payload components to ISS and store them on-orbit.

\section{Free Flyer Perspectives on Impacts}

Redesign changes to EURECA are expensive and time consuming. Program/shared hardware availability, such as EXPRESS pallet hardware, servicing panels and logistics carriers are critical to free-flyer servicing success. But, the benefits can be far-reaching, including commercialization of ISS, enhanced science missions, and providing a building block for exploration missions. 


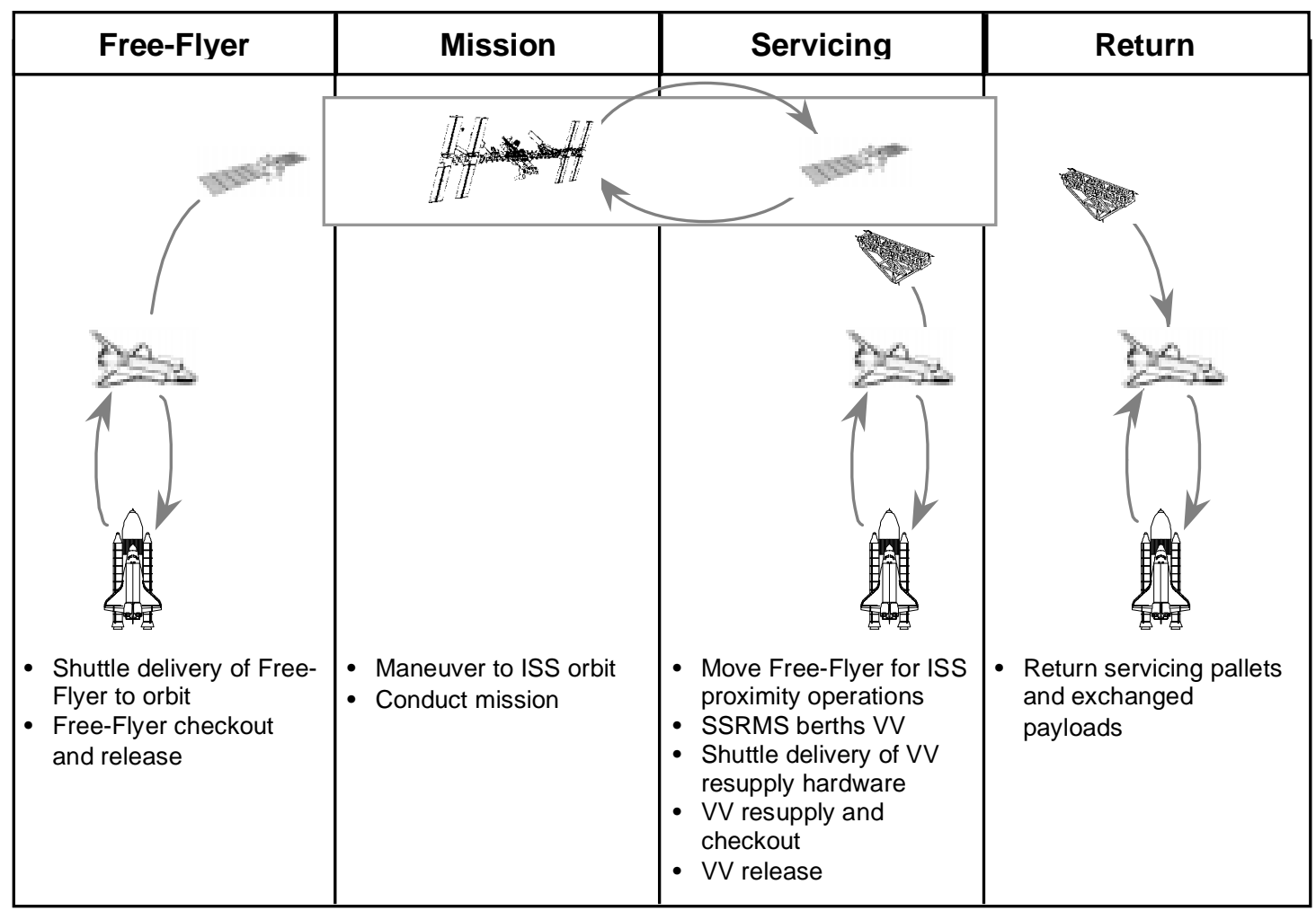

FIGURE 3. Free-Flyer Servicing Mission Scenario.

\section{IMPACTS TO THE INTERNATIONAL SPACE STATION}

A proposed design reference mission for free-flyer servicing at ISS is displayed in Figure 3. The scenario is a four step process that includes: the delivery of the free-flyer to orbit by the shuttle, free-flyer mission, servicing of the free-flyer at ISS, and free-flyer return to operation.

Servicing of Free-Flying spacecraft at the ISS will impact ISS operations and capabilities. The following sections define the areas of concern and the potential impacts.

\section{Locations on ISS for Free-Flyer Servicing}

Unpressurized free-flyers may be berthed to attached payload locations on the ISS truss; the Starboard-3 (S3) and Port-3 (P3) segments (ISS Program Office, 1998). Four attached payload sites are defined for S3, two for P3. The P3 locations are intended primarily for logistics stowage, however, they may be available to payloads on a noninterference basis. Current planning indicates potential over-subscription of these sites, however, other locations for free-flyer servicing may include the JEM Exposed Facility, the planned European Attached Facility, and additional sites on the truss.

\section{Free Flyer Capture and Berthing to ISS}

The Space Station Remote Manipulator System (SSRMS) would be needed for capturing free-flyers and berthing them to an attached payload site. Free-Flyers will need to have an SSRMS grapple fixture and the passive side of a PAS mechanism for berthing at the attached payload locations on the truss (ISS Payloads Office, 1998). A freeflyer will have to maintain its attitude and relative position in a defined grapple box for a minimum of 5 minutes, for capture by the SSRMS. 


\section{Free-Flyer Spacecraft Servicing}

ISS will need to provide an on-orbit re-fueling capability. Free-Flyers will need to be modified (or designed) for automated and/or EVA refueling operations. The preferred scenario is to change-out propellant tanks rather than conduct hazardous fuel transfer operations. If refueling is needed, however, the lessons learned from the Orbital Refueling System (ORS) experiment, flown on STS 41-G in 1984, and the Compton Gamma Ray Observatory (GRO) design should be applied.

\section{Free Flyer Payload Servicing and Change-out}

ISS will need to provide resources (SSRMS, EVA) for conducting on-orbit payload change-out and servicing. Free-Flyers should be modified (or designed) to accept EXPRESS Pallet Adapters (EPA) for payload change-out. EPAs are replaceable using the SSRMS and provide standardized interfaces (structural, power, and data). EVA would be used only in contingency situations. Support of automated payload checkout capabilities is also be needed for verification prior to release from ISS.

\section{Transportation and Stowage of Free-Flyer Consumables \& Payloads}

EXPRESS pallets and the SPACEHAB "Integrated Cargo Carrier" (ICC) could be used to transport consumables and hardware. Re-supply items would be delivered and stored on the ISS at the S3 or P3 attached payload locations prior to Free-Flyer berthing. In addition to S3 and P3, other potential locations for storage may include the JEM Exposed Facility, the planned European Attached Facility, and additional sites on the truss.

\section{Free-Flyer Servicing Impacts to Utilization.}

Free-flyer servicing activities would be limited to periods outside the ISS "quiescent" period, to prevent disturbance of the ISS microgravity environment. Loss of ISS attached payload accommodations, because of the servicing capability, would be offset by the additional payload accommodations provided by the free-flyers. The longer duration microgravity environments, flexibility of operations, enhanced pointing capability, altitude adjustment, and low contaminate levels provided by candidate free-flyers would also offset the loss of attached payload accommodations.

\section{CONCLUSIONS}

In order to provide servicing support to a wide range of free-flying "visiting vehicle" spacecraft, as shown in Figure 1, the ISS program will need to address the impacts discussed in the previous section. In particular, the locations for conducting servicing as well as stowage of consumables and equipment for change-out on ISS are critical for success. Also important is the definition of servicing activities and capabilities to be provided by ISS, in particular, for refueling operations. The associated costs will also need to be examined in detail to determine potential benefits and to encourage commercial participation. As discussed previously, while the implementation of a free-flyer servicing capability could be an expensive and time consuming prospect, the benefits can be farreaching, including commercialization of ISS, enhanced science missions, and providing a building block for exploration missions.

\section{ACKNOWLEDGMENTS}

The authors would like to acknowledge Mr. Lewis L. Peach, Director of Advanced Projects at NASA Headquarters, for his support in this study effort. 


\section{REFERENCES}

Daimler-Benz Aerospace, Space Infrastructure, EURECA User Guide, CD-ROM version, April 1996.

ISS Payloads Office, Attached Payloads Accommodations Team, World Wide Web page, 1998

http://iss-www.jsc.nasa.gov/ss/issapt/payofc/OZ3/att_accom.html

ISS Program Office, "Interface Definition Document (IDD) for International Space Station (ISS) Visiting Vehicles (VVs)", SSP 50235, July 31, 1998. 